\title{
A fotografia no estudo do eclipse lunar
}

\author{
The photograph in the study of the lunar eclipse
}

\author{
Alberto Bertuola*1, Kelmy Victória Romão ${ }^{1}$ \\ ${ }^{1}$ Instituto Federal de Educação, Ciência e Tecnologia de São Paulo - DME, Rua Pedro Vicente, 625, Canindé, São Paulo, SP, \\ Brasil
}

Recebido em 01 de Abril, 2017. Revisado em 29 de Maio, 2017. Aceito em 30 de Maio, 2017.

\begin{abstract}
Obtivemos neste trabalho um valor para o ângulo do cone de sombra da Terra estudando um eclipse lunar total de duração máxima. Assumimos conhecidos o valor do período orbital da Lua, a distância média Terra-Lua e o diâmetro equatorial da Terra. Essas quantidades foram combinadas com o valor do parâmetro geométrico, obtido a partir do tratamento de imagem da fotografia do eclipse. Os resultados foram comparados com os valores atuais dos diâmetros aparente da Lua e do Sol.
\end{abstract}

Palavras-chave: Eclipse da Lua, diâmetro aparente.

In this work we have acquired a value for angle of the cone of the Earth's shadow, by studing a total lunar eclipse of maximal duration. We have asssumed knowledge about the value the Moon's orbital period, the average distance Earth-Moon and the Earth's diameter. We have combined these quantities with a geometric parameter obtained through the image processing of the lunar eclipse photo. The results were compared with the current values of the apparent diameters of the Moon and the Sun.

Keywords: Eclipse of the Moon, apparent diameter.

\section{Introdução}

A determinação da distância absoluta da Terra à Lua estudando os eclipses [1] é um tradicional assunto abordado em livros textos de Física Básica 2, 3]. A partir de uma engenhosa triangulação do eclipse da Lua e de medidas observacionais de algumas grandezas, a distância Terra-Lua é obtida com sucesso. No entanto, algumas aproximações são adotadas, mesmo antes de efetuar completamente os cálculos. Por exemplo, adotar o mesmo valor, tanto para o diâmetro aparente da Lua, quanto para o ângulo do cone de sombra da Terra. Na realidade, o ângulo do cone de sombra assume um valor bem próximo ao do diâmetro aparente do Sol, quando este astro é observado de um ponto da superfície da Terra. Coincidentemente o diâmetro aparente médio da Lua é também quase igual ao diâmetro aparente médio do Sol. Para confirmar as afirmações anteriores consideramos neste trabalho, os valores numéricos atuais dos diâmetros aparentes do Sol e da Lua, que estão disponibilizados na página eletrônica oficial da NASA ${ }^{1}[4,5$. Após algumas transformações de unidades, esses valores são modificados em uma forma mais conveniente para as nossas investigações, conforme mostrados na tabela 1.

Chamamos a atenção para a última linha da tabela 2, cujo valor do diâmetro aparente médio da Lua é aquele

\footnotetext{
*Endereço de correspondência: acbertuola@gmail.com
}

${ }^{1}$ National Aeronautics and Space Administration. que servirá para as devidas comparações com os resultados obtidos neste trabalho.

Aristarco de Samos ( 300 A.C.) obteve as distâncias astronômicas Terra-Sol e Terra-Lua [6] em função do raio da Terra, estudando os eclipses solar e lunar. A mesma configuração geométrica do eclipse lunar usada por esse antigo astrônomo é utilizada aqui como base de estudo. A abordagem agora é um pouco diferente, ou seja, inserimos no modelo geométrico e cinemático, a participação de um elemento verdadeiramente moderno, desconhecido na época deste sábio: uma fotografia do eclipse lunar. As construções geométricas caracterizam o tratamento da imagem fotográfica, produzindo um valor numérico do parâmetro geométrico, que representará efetivamente a participação da fotografia neste estudo. Além disso, consideramos conhecidos, sem apresentar as incertezas experimentais, os valores do diâmetro da Terra, da distância média Terra-Lua e do período de revolução da Lua em torno da Terra. Todos esses valore 22 foram divulgados pela NASA e aqui alocados na tabela 2 .

A tabela 2 contém todos os valores daquelas grandezas relevantes para os propósitos deste trabalho. Alguns

${ }^{2} \mathrm{O}$ valor de uma grandeza experimental sempre deve ser representado por meio de um valor médio e uma incerteza experimental. Para os objetivos imediatos de comparação, adotaremos neste trabalho apenas os respectivos valores médios das grandezas. Um valor estimado para a incerteza experimental da medida do raio da Terra poderá ser encontrado na referência [7]. Um trabalho que confirma o valor do período de revolução da Lua é encontrado na referência 8. 
Tabela 1: Valores do tamanho aparente do Sol e da Lua.

\begin{tabular}{cccc}
\hline Diâmetro Aparente & Máximo $\left(^{\circ}\right)$ & Médio $\left.^{\circ}{ }^{\circ}\right)$ & Mínimo $\left(^{\circ}\right)$ \\
\hline Sol & 0,542 & 0,533 & 0,524 \\
\hline Lua & 0,491 & 0,527 & 0,548 \\
\hline
\end{tabular}

Tabela 2: Grandezas astronômicas conhecidas.

\begin{tabular}{lc}
\hline Grandeza astronômica & valor numérico \\
\hline Raio equatorial médio da Terra $(\mathrm{km})$ & $6,378 \times 10^{3}$ \\
\hline Distância média Terra-Lua $(\mathrm{km})$ & $3,844 \times 10^{5}$ \\
\hline Período sideral de revolução da Lua (dias) & 27,32 \\
\hline
\end{tabular}

comentários são necessários a respeito de cada grandeza astronômica utilizada. A primeira delas é o raio equatorial da Terra $\left(R_{T}\right)$, cujo valor foi determinado por Eratóstenes ( 225 A.C.), já com uma razoável precisão. A obtenção deste resultado foi uma realização notável, se levarmos em consideração que esse trabalho foi levado a cabo, por um físico da antiga biblioteca de Alexandria (Egito), em condição rudimentar quando comparada com a atual tecnologia. A distância da Terra-Lua $\left(d_{T L}\right)$ é obtida com grande precisão usando a emissão de um feixe de laser, a partir de um ponto na superfície da Terra. Após a reflexão do feixe na Lua, ele é captado novamente no ponto original de emissão, em um tempo muito bem cronometrado. O eclipse estudado neste trabalho, ocorreu no dia 15 de junho de 2011 e, nesse mesmo mês, a Lua alcançou em sua órbita, o periélio e o afélio 9 respectivamente nos dias 11 e 24 . Sabendo que a velocidade angular da Lua próxima do periélio é maior do que aquela, quando a Lua se encontra nos arredores do afélio, assumimos que na ocasião do eclipse, a Lua estava a uma distância média da Terra. O período sideral é aquele tempo decorrido, para a Lua percorrer uma órbita completa em torno da Terra. O período sinódico é o intervalo de tempo entre as duas fases consecutivas de Lua cheia. Não podemos afirmar com certeza qual o tempo considerado na antiguidade, mas neste trabalho, $T_{L}$ sempre representará o tempo sideral.

Admitimos conhecidas as medidas do valor da distância média Terra-Lua, do intervalo de tempo decorrido para a Lua percorrer uma distância igual ao seu próprio diâmetro e do valor numérico de um parâmetro geométrico, definido após um tratamento da imagem fotografada do eclipse. Usando os valores da tabela 2, calculamos o valor para o ângulo do cone de sombra da Terra, que foi comparado com o valor conhecido do diâmetro aparente da Lua (tabela 1).

O eclipse lunar [10] escolhido para o estudo é aquele cuja principal característica é ser um eclipse de maior tempo de duração. Ele será conveniente para uma investigação, pois, apropriadamente, a Lua atravessa o cone de sombra quase que diametralmente, conforme mostrado na figura 1 .

O desenho da figura 1 é uma composição de fotos da Lua, desenvolvendo seu movimento através do cone de sombra da Terra. A Lua inicia seu percurso para o in-

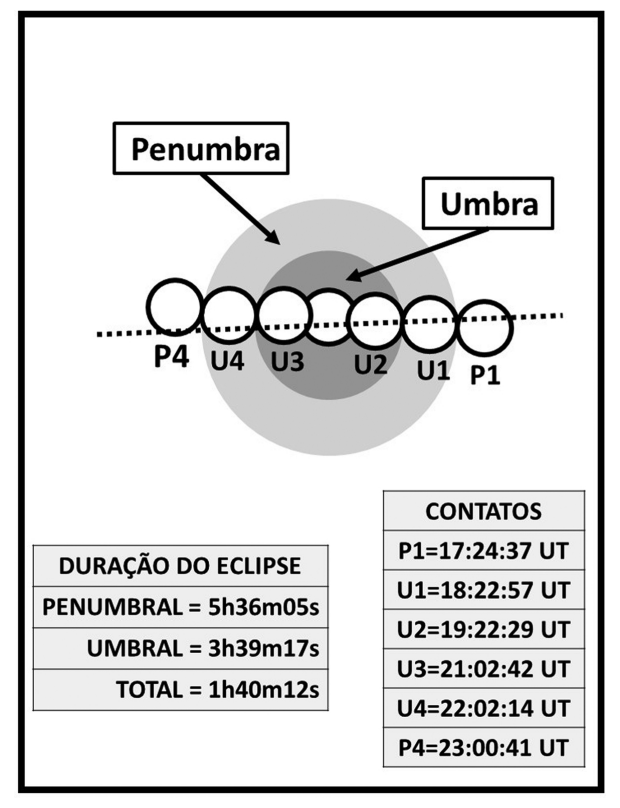

Figura 1: Dados divulgados pela NASA para o eclipse total da Lua ocorrido no dia 15 de junho de 2011.

terior do cone na posição U1, denominada de primeiro contato. O segundo contato U2 é aquela posição, quando a Lua já percorreu uma distância igual ao seu próprio diâmetro, se escondendo inteiramente dentro da sombra. O intervalo de tempo para essa ocorrência é calculado por meio da igualdade $\Delta t=t_{U_{2}}-t_{U_{1}}$, ou seja, é a diferença entre os horários dos dois contatos iniciais. Calcular esse intervalo de tempo registrando os horários $t_{U_{2}}$ e $t_{U_{1}}$ é uma característica própria de um eclipse de duração total máxima. Esse intervalo pode ser calculado alternativamente usando $\Delta t=t_{U_{4}}-t_{U_{3}}$, em que $t_{U_{4}}$ e $t_{U_{3}}$ são os respectivos horários dos contatos U3 e U4, que ocorrem no final do eclipse. Quando a Lua atingir o terceiro contato U3, ela terá percorrido uma corda de comprimento, quase igual ao diâmetro da secção transversal plana e circular do cone de sombra. O tempo gasto para este percurso é calculado usando $\Delta t_{e}=t_{U_{3}}-t_{U_{1}}$. Os valores para os horários de contatos $\left(t_{U_{1}}, t_{U_{2}}, t_{U_{3}}, t_{U_{4}}\right)$ estão apresentados na parte inferior direita da figura 1. Os valores numéricos de $\Delta t$ e $\Delta t_{e}$ para esse peculiar eclipse serão inevitavelmente usados mais adiante. Eles foram calculados rapidamente e deixados a disposição na tabela 3 .

Os resultados apresentados na tabela 3 são frutos das importantes e cuidadosas atividades experimentais no estudo do eclipse.

Tabela 3: Intervalos de tempo observados no eclipse lunar.

\begin{tabular}{lc}
\hline Tempo & valor numérico $(\min )$ \\
\hline$\Delta t=t_{U_{2}}-t_{U_{1}}$ & 59,53 \\
\hline$\Delta t=t_{U_{4}}-t_{U_{3}}$ & 59,53 \\
\hline$\Delta t_{e}=t_{U_{3}}-t_{U_{1}}$ & 159,75 \\
\hline
\end{tabular}


Apresentamos na seção imediatamente a seguir: a fotografia do eclipse, acompanhada de um tratamento geométrico na sua imagem; um conjunto de dados experimentais produzidos por essas construções e a definição do parâmetro geométrico. Na seção seguinte, explicamos o envolvimento da triangulação do eclipse lunar com a cinemática, resultando a importante conexão entre os parâmetros físico e geométrico na forma de equações, que são usadas para calcular o valor do ângulo do cone de sombra. Os resultados obtidos são apresentados exclusivamente em uma seção consecutiva. Encerramos este artigo com uma seção final explicativa.

\section{Fotografando o Eclipse Lunar}

O eclipse lunar do dia 15 de junho de 2011 foi muito bem estudado [11] e rigorosamente documentado de diversas maneiras. Entre todos os sucessivos quadros de um vídeo deste eclipse, selecionamos um com boa qualidade de imagem. $\mathrm{O}$ quadro escolhido é mostrado na figura $2 \mathrm{com}$ todas as construções geométricas inseridas.

A figura 2 mostra dois pares de anéis circulare ${ }^{3}$ cada um deles englobando as respectivas fronteiras, da Lua e da secção transversal circular do cone de sombra da Terra. Essas construções geométricas são feitas na prática, com finos traços e cuidadosos ajustes. Nessa mesma figura, em cada região indicada por $I, I I, I I I$ e $I V$, dois pontos são determinados pelo cruzamento dos círculos concêntricos e a extremidade de uma reta vertical diametral. Por exemplo, quatro valores de distâncias entre os pontos das regiões $I$ e $I I$ são obtidos, combinando os pontos destas

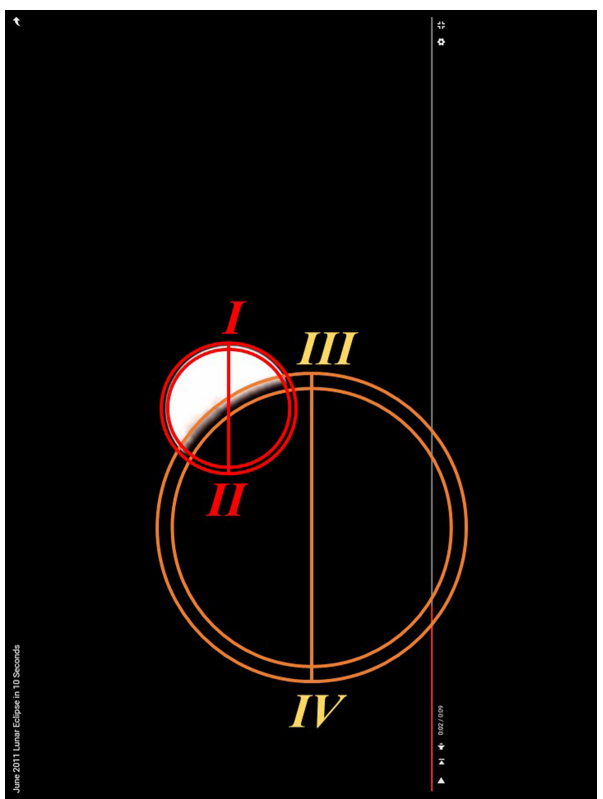

Figura 2: Um quadro fotográfico do eclipse lunar.

\footnotetext{
${ }^{3} \mathrm{Na}$ imagem não se verifica uma borda circular perfeita para Lua e nem para a fronteira da projeção do cone de sombra sobre ela. Por isso, usamos dois círculos concêntricos para envolver as fronteira e levar em consideração as deformações registradas na foto.
}

regiões por meio de subtrações. As distâncias obtidas servem de estimativas para o tamanho do diâmetro da Lua $\left(D_{L}\right)$ na figura 2. Da mesma forma, quatro distâncias entre os pontos das regiões $I I I$ e $I V$ são relativos ao diâmetro do cone de sombra $\left(D_{C}\right)$. Um dos tratamentos de imagem realizado, produziu os valores numéricos apresentados na tabela 4 .

Os valores conhecidos dos diâmetros das imagens da Lua e da secção do cone de sombra são relacionados pela definição do parâmetro geométrico $(\eta)$ na forma da razão

$$
\eta=\frac{D_{L}}{D_{C}}
$$

Por construção, os valores dos diâmetros $D_{L}$ e $D_{C}$ são independentes entre si. Quando são combinados pela equação (1), para cada tratamento de imagem realizado, dezesseis valores são obtidos para o parâmetro geométrico $(\eta)$. A estatística do parâmetro geométrico pode ser enriquecida realizando mais tratamentos de imagem do eclipse lunar.

\section{Cinemática e a Triangulação do Eclipse}

A triangulação do eclipse lunar adotado neste trabalho está esboçada na figura 3 .

A figura 3 mostra um plano vertical que secciona transversalmente o cone de sombra. Nele estão contidos uma secção equatorial da Terra e o vértice $(V)$ do cone de sombra, que tem uma certa abertura $(\alpha)$. Na triangulação plana as igualdades $\overline{O P}=R_{T}$ e $\overline{O Q}=d_{T L}$ são identificadas sem dificuldades. O deslocamento da Lua durante o eclipse sofre uma conveniente aproximação,

Tabela 4: Tamanhos das imagens no eclipse lunar.

\begin{tabular}{cc}
\hline$D_{L}$ & $D_{C}$ \\
\hline 3,1 & 9,8 \\
\hline 4,1 & 10,1 \\
\hline 4,1 & 10,1 \\
\hline 4,3 & 10,4 \\
\hline
\end{tabular}

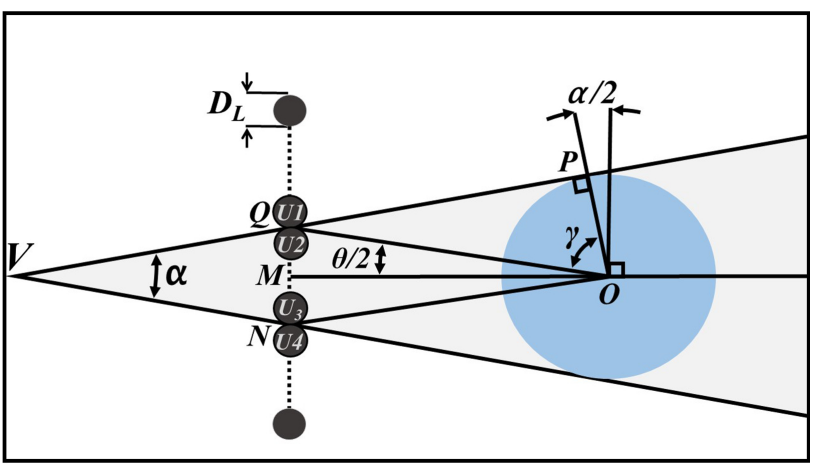

Figura 3: Triangulação no fenômeno do eclipse lunar. As posições de contatos da Lua com a umbra são representadas por $(\mathrm{U} 1, \mathrm{U} 2, \mathrm{U} 3, \mathrm{U} 4)$. 
substituindo a pequena trajetória curva $Q N$, por um seguimento de reta $\overline{Q N}$. Fazendo uso da trigonometria básica nos triângulos retângulos $\widehat{O P Q}$ e $\widehat{O M Q}$ e, relacionando todos ângulos na região do ponto $O$, obtivemos o sistema de equações geométricas

$$
\left\{\begin{array}{l}
\cos (\gamma)=\frac{R_{T}}{d_{T_{L}^{L}}} \\
\sin \left(\frac{\theta}{2}\right)=\frac{D_{C}}{2 d_{T L}} \\
\frac{\theta}{2}+\gamma+\frac{\alpha}{2}=\frac{\pi}{2}
\end{array} .\right.
$$

Os dados da tabela 3 revelam que a Lua percorre distâncias equivalentes ao seu próprio diâmetro, em mesmo intervalos de tempo, em relação à uma precisão considerada, seja eles medidos no início ou no final do eclipse. Esta base experimental indica que o movimento da Lua em torno da Terra, na escala de tempo de duração do eclipse é aproximadamente circular uniforme. Se a velocidade angular da Lua é constante durante o fenômeno, então o parâmetro geométrico (1) pode ser reescrito na forma

$$
\eta=\frac{\Delta t}{\Delta t_{e}} .
$$

A invariância da velocidade angular da Lua em torno da Terra, durante a ocorrência do eclipse, permite escrever também

$$
D_{C}=\left(\frac{2 \pi}{T_{L}} d_{T L}\right) \Delta t_{e} .
$$

Combinando (4), (3) e (2) e adicionando uma certa quantidade de manipulações matemáticas, obtém-se

$$
\alpha=2\left[\sin ^{-1}\left(\frac{R_{T}}{d_{T L}}\right)-\sin ^{-1}\left(\frac{\delta}{\eta}\right)\right],
$$

em que $\delta=\frac{\pi \Delta t}{T_{L}}$ é um parâmetro físico, definido naturalmente no desenvolvimento das contas. A equação (5) é aquela que permite determinar o valor para a abertura do cone de sombra.

\section{Resultados e Análises}

Considerando os valores dos diâmetros obtidos pelos autores no tratamento da imagem, obtém-se um conjunto de valores numéricos para o ângulo do cone de sombra, que foram distribuídos na forma de um histograma, conforme é mostrado na figura 4 .

Os valores mais prováveis apontados pelo histograma da figura 4, se assemelham aos típicos valores para o diâmetro aparente da Lua, quando comparados com aqueles apresentados na tabela 1.

Dois resultados numéricos experimentais para o ângulo do cone estão divulgados na tabela 5 .

O primeiro valor numérico na tabela 5 é uma estimativa do ângulo do cone, que é calculado por meio das equações (3) e (5) e dos valores nas tabelas 1 e 3. Sabendo que o valor do ângulo do cone de sombra deve ser menor que o diâmetro aparente da Lua, então este valor parece

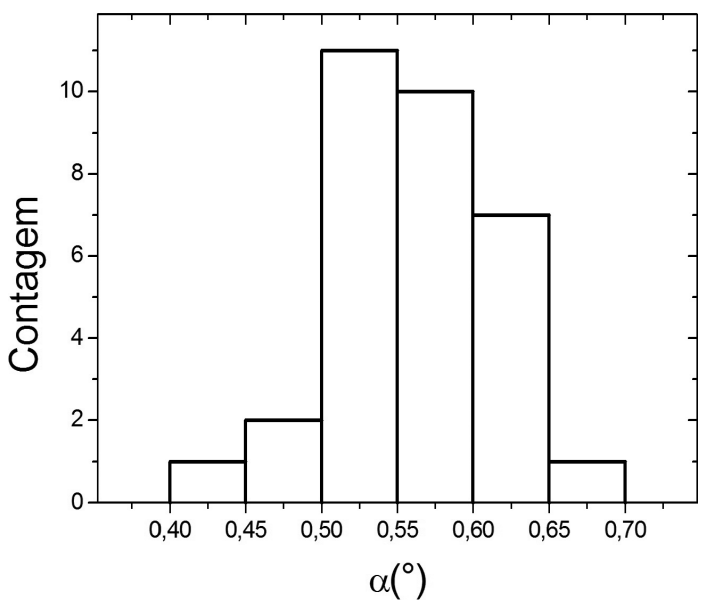

Figura 4: Histograma dos valores do ângulo do cone de sombra.

Tabela 5: Valores experimentais do ângulo do cone de sombra.

$\frac{\alpha\left(^{\circ}\right)}{0,46}$

bem razoável deste ponto de vista, mas certamente subestimado. O segundo valor foi obtido usando o parâmetro geométrico definido na equação (1), cujo resultado experimental é um valor médio $\left(0,56^{\circ}\right)$ muito próximo daquele atribuído ao diâmetro aparente médio da Lua (ou do Sol) na tabela 1. A barra de erro que acompanha este valor, emerge principalmente das próprias construções geométricas. A pouca nitidez na fronteira do cone de sombra é opositora ao ajuste perfeito. Na prática, após várias construções, o valor médio do ângulo resulta maior do que aquele da tabela 1 . A incidência da luz do Sol sobre a superfície esferoidal da Lua pode estar alterando localmente a curvatura real da fronteira do cone. Por exemplo, se a curvatura do cone de sombra sofresse um aumento local, o diâmetro do cone de sombra diminuiria. Pela equação (1), o parâmetro geométrico seria amplificado e de acordo com a equação (5), o ângulo do cone de sombra teria seu valor levemente superestimado. A figura 5 mostra duas imagens em dois momentos distintos do eclipse lunar, quando a Lua está adentrando à região escura, para o interior do cone de sombra da Terra.

Conforme a figura 5, a melhor nitidez na fronteira da sombra é aquela em que metade da Lua está iluminada. Essa foto é aquela mais adequada para o estudo em questão.

\section{Comentário Final}

Para estudar o eclipse lunar usando uma fotografia desse fenômeno, recorremos ao estudo do eclipse lunar total de duração máxima. Nesse tipo de eclipse, o intervalo de tempo entre os dois contatos no início do eclipse, coincide com aquele tempo em que a Lua percorre uma distância igual ao seu próprio diâmetro. A medida experimental 


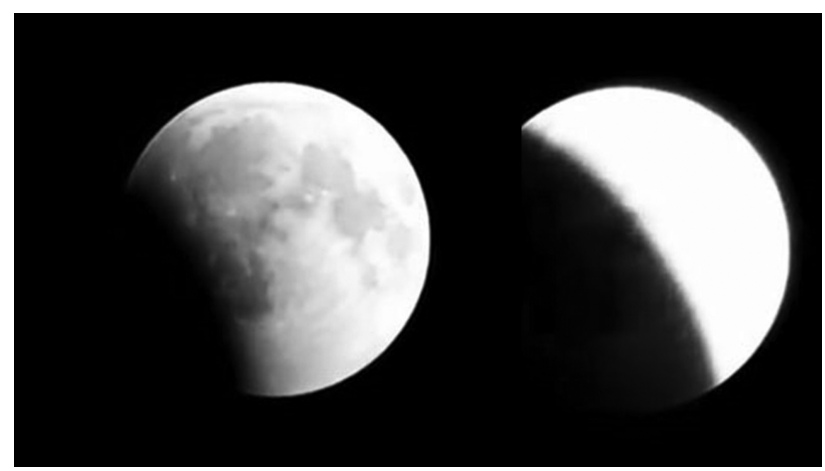

Figura 5: Dois momentos no eclipse da Lua.

do valor deste intervalo de tempo é realizada com grande precisão. Por outro lado, os valores do diâmetro da Lua e do diâmetro do cone de sombra são coletados diretamente da fotografia do eclipse, com suas prévias construções geométricas. Eles são substituídos na razão matemática (1) e o valor numérico do parâmetro geométrico é obtido. Em seguida, todos os valores pertinentes são substituídos na equação (5), que é obtida de uma triangulação plana combinada com um modelo cinemático simples. Finalmente o ângulo do cone de sombra da Terra é calculado e o valor encontrado neste trabalho vale $\alpha=0,56^{\circ} \pm 0,05^{\circ}$, mostrando um surpreendente e agradável resultado, ou seja, o valor do ângulo do cone de sombra da Terra obtido no fenômeno do eclipse lunar é, dentro do intervalo de erro estimado, igual aos valores dos diâmetros aparentes da Lua e do Sol. Este resultado satisfaz completamente as nossas expectativas didáticas iniciais.

\section{Agradecimentos}

K. V. O. Romão agradece o CNPq pelo apoio financeiro. Os autores dirigem um especial agradecimento ao Departamento de Mecânica (IFSP-Campus São Paulo).

\section{Referências}

[1] L.B.F. Clauzet e L. Sodré Jr., Revista Brasileira de Ensino de Física 2, 64 (1980).

[2] A.P. French, Newtonian Mechanics (Norton, New York, 1971).

[3] H.M. Nussenzveig, Curso de Fúsica Básica, 1- Mecânica (Editora Edgard Blücher Ltda, São Paulo, 2002), 4ª ed.

[4] https://nssdc.gsfc.nasa.gov/planetary/ factsheet/sunfact.html, acesso em 13/10/2016.

[5] https://nssdc.gsfc.nasa.gov/planetary/ factsheet/moonfact.html, acesso em 10/10/2016.

[6] T.B. de Oliveira, V.T. Lima e A.C. Bertuola, Revista Brasileira de Ensino de Física 2, 2304 (2016).

[7] A.J. de Jesus Santos, M.R. Voelzke e M.S.T. de Araújo, Caderno Brasileiro de Ensino de Física 3, 1137 (2012).

[8] A.P. Tonel e G.F. Marranghello, Revista Brasileira de Ensino de Física 2, 2310 (2013).

[9] https://www.vercalendario.info/pt/lua/brasilmes-junho-2011.html, acesso em 10/10/2016.
[10] https://eclipse.gsfc.nasa.gov/LEplot/ LEplot2001/LE2011Jun15T .pdf , acesso em 10/10/2016.

[11] https://www . youtube.com/watch?v=VmTAF91EWp8, acesso em 11/10/2016. 